Supporting Information for

\title{
Copper(III) and Vanadium(IV)-Oxo Corrolazines
}

Joseph P. Fox, ${ }^{\dagger}$ Bobby Ramdhanie, ${ }^{\dagger}$ Adelajda A. Zareba, ${ }^{\dagger}$ Roman S. Czernuszewicz, ${ }^{\dagger}$ and David P. Goldberg, ${ }^{*} \dagger$

${ }^{\dagger}$ Department of Chemistry, The Johns Hopkins University, Baltimore, Maryland, 21218 and

${ }^{*}$ Department Chemistry, University of Houston, Houston, Texas 77204-5003

\section{Contents}

Figure S1. ${ }^{1} \mathrm{H}$ NMR of $(\mathrm{TBP}){ }_{8} \mathrm{CzCu}^{\mathrm{III}}$ at $300 \mathrm{~K}$ ( $\mathrm{s}=$ solvent peaks, $*=$ impurity). The slight broadening observed in the aromatic region as compared to the $400 \mathrm{~K}$ spectrum shown in Figure 3 (see main text) is likely due to increased aggregation at this temperature.

Figure S2. EPR simulation of (TBP $)_{8} \mathrm{Cz}(\mathrm{H}) \mathrm{V}^{\mathrm{IV}} \mathrm{O}$.

Figure S3. EPR simulation of $(\mathrm{TBP}){ }_{8} \mathrm{CzCu}^{\mathrm{III}}$. 


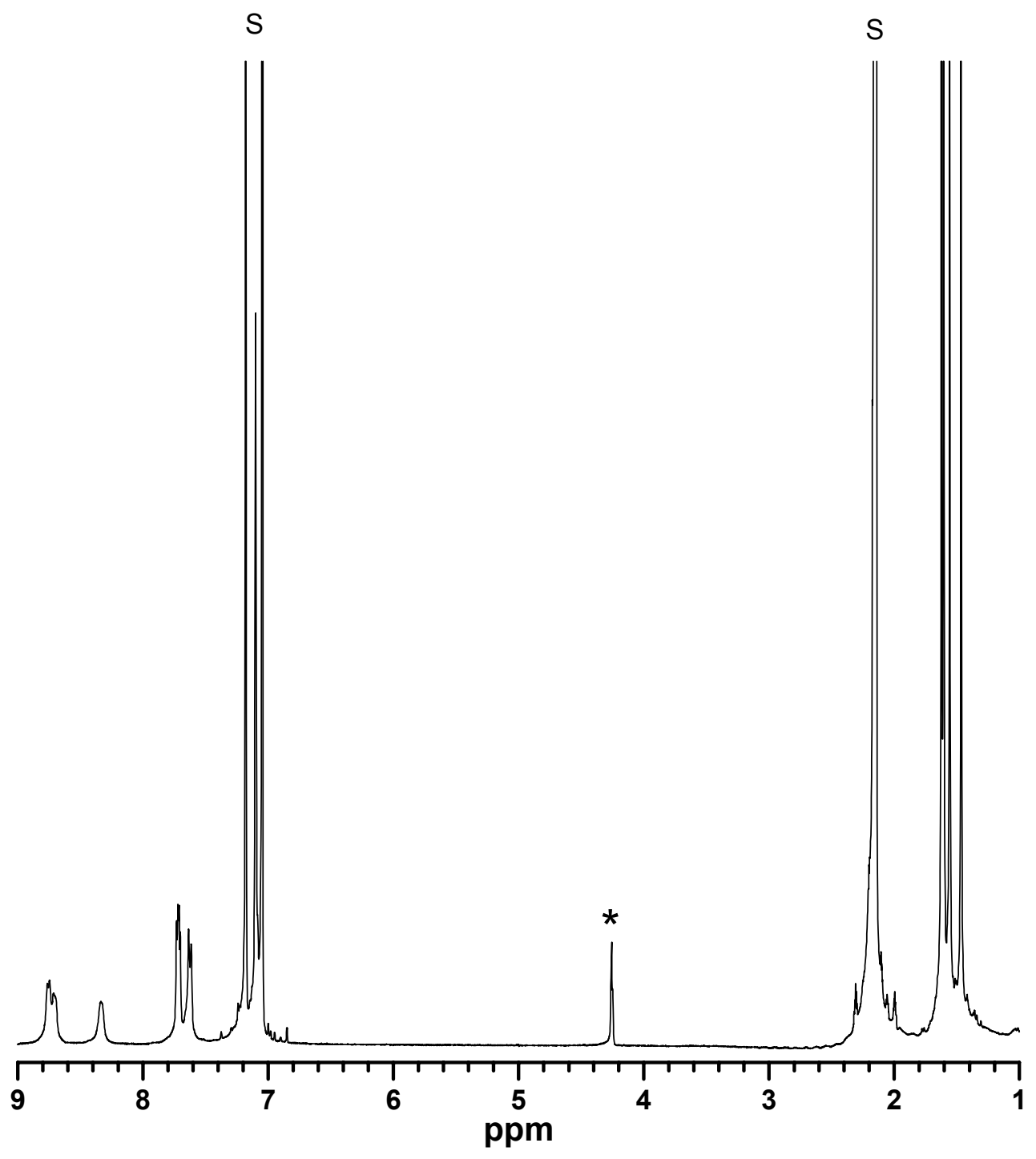

Figure S1. Fox, Ramdhanie, Zareba, Czernuszewicz, and Goldberg. 


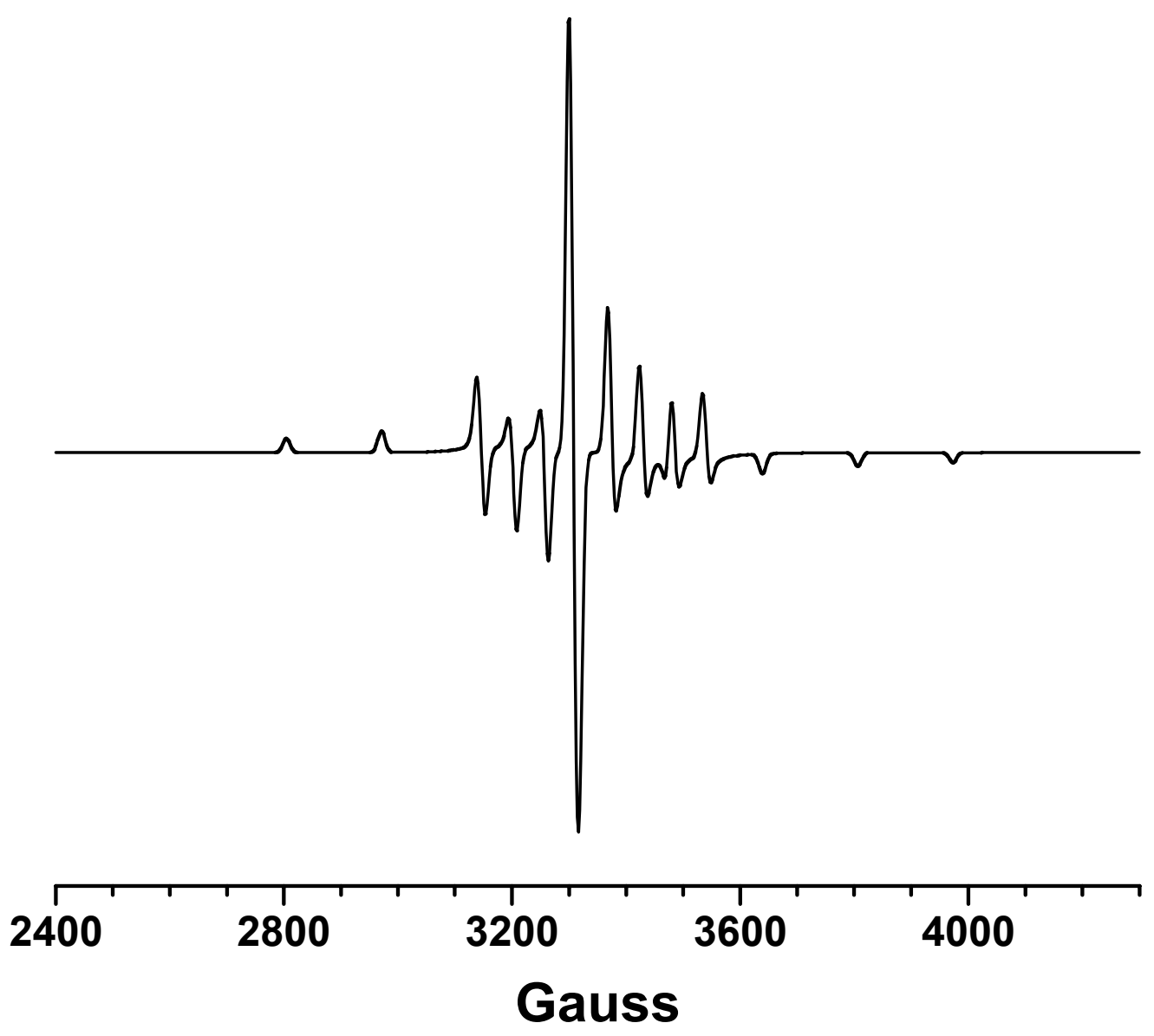

Conditions: frequency, $9.308 \mathrm{GHz}$; incident microwave power, $63.46 \mathrm{~mW}$; modulation frequency, $100 \mathrm{kHz}$; modulation amplitude, $10.0 \mathrm{G}$; receiver gain, $7.25 \mathrm{~dB}$; line width, $15 \mathrm{G}$.

Figure S2. Fox, Ramdhanie, Zareba, Czernuszewicz, and Goldberg. 


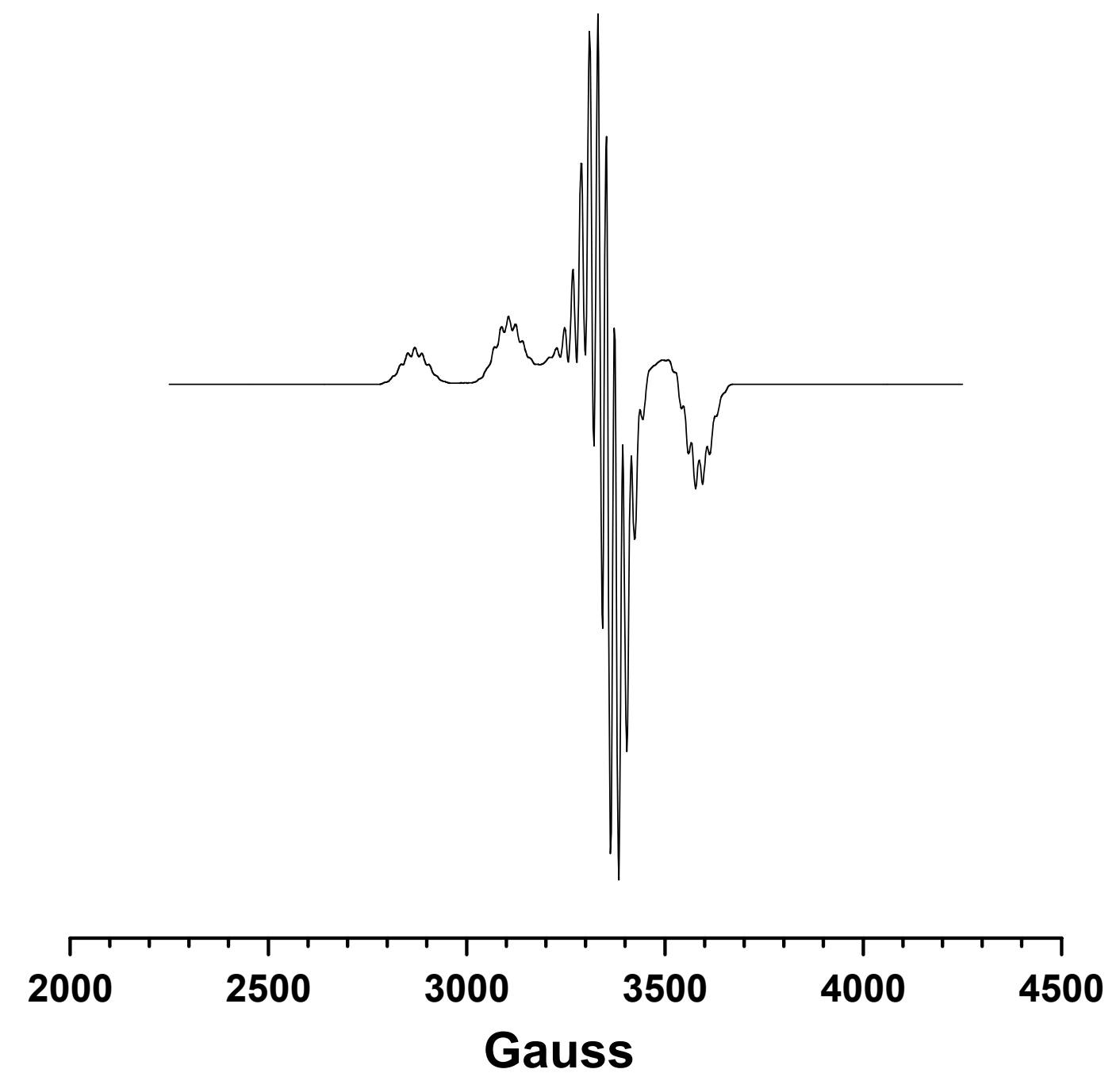

Conditions: frequency, $9.305 \mathrm{GHz}$; incident microwave power, $63.46 \mathrm{~mW}$; modulation frequency, $100 \mathrm{kHz}$; modulation amplitude, $10.0 \mathrm{G}$; receiver gain, $1.81 \mathrm{~dB}$; line width, $14 \mathrm{G}$.

Figure S3. Fox, Ramdhanie, Zareba, Czernuszewicz, and Goldberg. 